\title{
SIMULATION OF CONTINUOUSLY DEFORMING PARABOLIC PROBLEMS BY GALERKIN FINITE-ELEMENTS METHOD
}

\author{
YAHIA S. HALABI \\ Department of Computer Science \\ Jordan University \\ Amman, Jordan
}

(Received August 6, 1985)

ABSTRACT. A general numerical finite element scheme is described for parabolic problems with phase change wherein the elements of the domain are allowed to deform continuously. The scheme is based on the Galerkin approximation in spare, and finite difference approximation for the time derivatives. The numerical scheme is applied to the two-phase Stefan problems associated with the melting and solidification of a substance. Basic functions based on Hermite polynomials are used to allow exact specification of flux-latent heat balance conditions at the phase boundary. Numerical results obtained by this scheme indicates that the method is stable and produces an accurate solutions for the heat conduction problems with phase change; even when large time steps used. The method is quite general and applicable for a variety of proilems involving transition zones and deforming regions, and can be applied fur one multidimensional problems.

KEY WORDS AND PHRASES. Stefan problems, Hermite, transition zones, muving boundary, Gaterkin approximation.

1980 AMS SUBJECT CLASSIFICATION CODE. 65N30.

1. INTRODUCTION.

Application of the finite element method to transport problems is now well established, and several valuable texts and compilations are currently available. The use of Galerkin method in conjuction with the finite element technique has significantly extended its scope such that a broad class of transient field problems can now be approached confidently with finite elements, particularly, for problems allowing continuous mesh deformation, which result that a moving boundary lies on element boundaries, Halabi [1]. The finite element method has proven to be valuable in these types of problems. It provides a mechanism for generating difference equations on a non-uniform mesh and allows the use of higher order elements in regions where they are suited to the physics of the problem.

Numerous solutions for Stefan problems have been reported, and several review articles are available [2]. Crank [3] gives a sufficient ar count of finite differeme 
method, indicating that, in general, both fixed and deforming grids have been used. Applications are largely confined to one-dimensional situations and frequently lack generality. The isotherm migration method [4] is in a sense a hyprid, in so far as the roles of the dependent and one of the independent space variable are interchanged, allowing a fixed grid to be created in the temperature domain. Among the moving grid approaches, a common problem would appeat to be the distorted mesh which would result in higher dimensional situations. Comini et al. [5] uses a fixed mesh approach and finite width transition zone. Latent heat effects in the transition zone were a counted for, by a special device (the 'apparent heat capacity' approach). Morgan et al. [6] have reported improvements of this method.

Herein, a general Galerkin finite element approach will be applied. In this approach, the choice of integration scheme in time is left to user, it is not dictated by the moving-mesh feature. The integration in time may thus be chosen to suit the details of a specific problem. This added flexibility is viewed as a potential advantage for moving boundary simulation in general.

2. PHYSICAL AND MATHEMATICAL MODEI.

The proposed model in this researh, described an internal transition zone 'internal moving boundary', changing primarily in location, as a function of time. This transition zone occurs between the frozen and unfrozen zones. As freezing proceeds with time, the location of the transition zone changes, and consequently, an important quantities change rapidly across it. The complexity in these types of problems arises on the mechanism of generating the governing difference equations on the non-uniform mesh of the domain of definition. In addition, a great numerical details might be required to describe the expected steep or mild gradients in temperature and other descriptive parameters across a frozen layer.

The model describes the freezing process, which is a two-phase stefan probe $1 \mathrm{~m}$. The domain $D$ is divided into $D_{1}$ and $D_{2}$ in which;

$$
\begin{aligned}
& \mathrm{D}=\left(\mathrm{D}_{1} \mathrm{ur}_{2}\right)+\mathrm{S} \text {; } \\
& \mathrm{D}_{1} \cap \mathrm{D}_{2}=\emptyset
\end{aligned}
$$

and $S$ is the internal moving boundary as shown in Figure (1).

The governing equations for this model are

$$
\begin{array}{ll}
\mathrm{C}_{1} \frac{\partial \mathrm{H}}{\partial \mathrm{t}}=\nabla \cdot\left(\mathrm{K}_{1} \nabla \mathrm{H}\right) & \text { in } \mathrm{D}_{1} \\
\mathrm{C}_{2} \frac{\partial \mathrm{H}}{\partial \mathrm{t}}=\nabla \cdot\left(\mathrm{K}_{2} \nabla \mathrm{H}\right) & \text { in } \mathrm{D}_{2}
\end{array}
$$

where $\left(\mathrm{C}_{1}, \mathrm{~K}_{1}\right),\left(\mathrm{C}_{2}, \mathrm{~K}_{2}\right)$ are the volumetric heat capacity and thermal conductivity of the frozen (unfrozen) portion of the medium, and $H$ denotes the temperature.

At the internal moving boundary, the temperature is a constant (the freezing temperature) and the velocity of the moving boundary is given by the boundary condition,

$$
\mathrm{L} \frac{\mathrm{ds}}{\mathrm{dt}}=\mathrm{K}_{1} \nabla \mathrm{H}(\mathrm{s})-\mathrm{K}_{2} \nabla \mathrm{H}(\mathrm{s})
$$

in which, $s$ : is the location of a given point on $S$, and $L$ is the volumetric latent heat of fusion of the soil-water mixture. 
When the temperature of the unfrozen material, is effectively constant throughout the freezing temperature, the problem reduces to the one-phase Stephan problem:

$$
\begin{aligned}
& \text { C } \frac{\partial H}{\partial t}=\nabla \cdot K \nabla H \\
& L \frac{d s}{d t}=K \nabla H(s)
\end{aligned}
$$

\section{NUMERICAL FORMULATION.}

The numerical approximation of this problem is derived from the general formulation of Galerkin finite element equations, with the extension to include the effects of deforming mesh by the addition of a single extra 'convective' term in the weighted residual equations. Basis functions derived from Hermite polynomials are used to stimulate this problem. They appear to offer accuracy and efficiency competitive with or superior to other basis function for the same computational effort, and have the particular attraction that gradient boundary conditions may be specified exactly.

In the presence of node motion, the finite element basis functions become implicit functions of time, $\emptyset(x, t)$, and the approximate solution can be written as:

$$
H(x, t)=\sum_{i} a_{i}(t) \emptyset_{i}(x, t)
$$

where $a_{1}(t)$ are the coefficients to be determined, and $\emptyset_{i}(x, t)$ are the Hermite basis functions. Consequent1y one can write

$$
\frac{\partial H}{\partial t}(x, t)=\frac{d a_{i}(t)}{d x} \emptyset(x, t)+a_{i} \frac{\partial \emptyset_{i}(x, t)}{\partial t}
$$

If $x$ is expressed as $x_{i} \xi_{i}$ using the directional function $\xi_{i}$ at each $x_{i}$, then the following is simply obtained,

$$
\frac{\partial \emptyset_{i}(x, t)}{\partial t}=-\frac{d x}{d t} \cdot \nabla \emptyset_{i}(x, t)
$$

in which $\mathrm{dx} / \mathrm{dt}$ represents the mesh deformation velocity which is continuous throughout the spatial domain, while the minus sign is due to the decrease in mesh space corresponding to a fixed origin in the unfrozen area, and $\emptyset(x, t)$ maps $R^{n}$ into $R^{n+1}$. Consequently, equation (3.2) becomes,

$$
\frac{\partial H(x, t)}{\partial t}=\emptyset_{i}(x, t) \frac{\partial a_{i}(t)}{\partial t}-a_{i}(t)\left(\frac{d x}{d t} \cdot \nabla \emptyset(x, t)\right)
$$

which can be written as,

$$
\frac{\partial H(x, t)}{\partial t}=\emptyset_{i}(x, t) \frac{\partial a_{i}(t)}{\partial t}-\frac{d x}{d t} \cdot \nabla H(x, t)
$$

Following the Galerkin approximation technique, assuming the representation of $H$ as in equation (3.1), and substituting into equation (2.6), then after taking the scalar product of $\emptyset_{i}$ with the resulting equation, the main equation can be written as

$$
\left(\emptyset_{i}, C \emptyset_{j}\right) \frac{\mathrm{da} j}{\mathrm{dt}}-\left(\emptyset_{i}, C \frac{\mathrm{dx}}{\mathrm{dt}} \cdot \nabla \emptyset_{j}\right) a_{j}+\left(\nabla \emptyset_{i}, K \nabla \emptyset_{j}\right) a_{j}-a_{j} \int_{\Gamma} n \cdot \emptyset_{i} k \nabla \emptyset_{j} d \Gamma=0
$$

where

$$
\left(\emptyset_{i}, \emptyset_{j}\right)=\int_{R} \emptyset_{i} \emptyset_{j} d R
$$

and $\Gamma(t)$ is the boundary of $R$. 
The potential advantage of this approach, is the choice of integration scheme in time, which is left to the user. The general approach is by applying equation (3.6) at time $t^{*}$, where

$$
t^{*}=t^{n}+\varepsilon\left(t^{n+1}-t^{n}\right), \quad 0<\varepsilon<1
$$

and using the following approximations,

$$
\begin{aligned}
& \frac{d a_{j}\left(t^{*}\right)}{d t} \approx \frac{\left.a_{j}^{n+1}-a_{j}^{n}\right)}{t^{n+1}-t^{n}} \\
& a_{j}\left(t^{*}\right) \approx \varepsilon a_{j}^{n+1}+(1-\varepsilon) a_{j}^{n}
\end{aligned}
$$

then equation (3.6) can be written as,

$$
\left[A_{i j}+\varepsilon B_{i j}\right] a_{j}^{n+1}=\left[A_{i j}+(\varepsilon-1) B_{i j}\right] a_{j}^{n}
$$

in which the coefficient matrices $A_{i j}$ and $B_{i j}$ have to be determined taking into consideration the time dependence of $\mathrm{dx} / \mathrm{dt}$ and the region of integrations to be performed. The solution of equation (3.11) yields to complete determination for the unknown coefficient vector $\vec{a}(t)$, which in turn, can be used to determine the temperature profile in any predefined region.

4. NUMERICAL SOLUTIONS.

The problem to be considered is a two-phase stefan problem in which freezing proceeds from the region into the medium which is bounded by fixed constant temperature boundaries;

$$
\begin{array}{ll}
\mathrm{H}=\mathrm{H}_{\mathrm{S}} & , \mathrm{x}=\mathrm{O} \\
\mathrm{H}=\mathrm{H}_{1} & , \mathrm{x}=\mathrm{x}_{1}, \mathrm{H}_{\mathrm{s}}<0<\mathrm{H}_{1} \\
\mathrm{H}=\mathrm{H}_{1} & , \mathrm{t}=0
\end{array}
$$

in which $\mathrm{H}_{\mathrm{S}}$ and $\mathrm{H}_{1}$ is the temperature of the frozen (solid) and unfrozen (1iquid) regions. The temperature of liquid phase is initially above the freezing point. Consequently, equation (2.3) applies over the frozen region $0<x<s(t)$, and equation (2.4) applies over the unfrozen region $s(t)<x<x_{1}$. Boundary and initial conditions are as in equation (3.12). The motion of the internal phase boundary $s(t)$ is given in equation (2.5); temperature is zero on this boundary.

Numerical solution in the liquid and solid phases can be approached as two separate heat transfer problems, coupled only by the moving boundary conditions which can be written as,

$$
\mathrm{L} \frac{\mathrm{ds}}{\mathrm{dt}}=\mathrm{K}_{1} \mathrm{H}_{\mathrm{s} 1}-\mathrm{K}_{2} \mathrm{H}_{11}
$$

where $\mathrm{H}_{\mathrm{Sl}}$, $\mathrm{H}_{11}$ are that coefficients corresponding to the frozen and unfrozen phase temperature gradient at the phase change boundary. The numerical approximation of equation (3.13) is.

$$
s^{n+l}=s^{n}+\frac{\Delta t}{L}\left(K_{1} \hat{H}_{s l}-K_{2} \hat{H}_{1 l}\right)
$$

where, 


$$
\begin{aligned}
& \widetilde{\mathrm{H}}_{\mathrm{s} 1}=\theta \mathrm{H}_{\mathrm{s} 1}^{\mathrm{n}+1}+(1-\theta) \mathrm{H}_{\mathrm{s} 1}^{\mathrm{n}} \\
& \tilde{\mathrm{H}}_{11}=\theta \mathrm{H}_{11}+(1-\theta) \mathrm{H}_{11}
\end{aligned}
$$

This problem is solved using predictor-corrector scheme. The selection of $\theta=0.54$ for the satisfaction of moving boundary condition, and $\varepsilon=0.62$ for the temperature solution would appear to be the ideal choice. The present formulation allows the user an additional valuable numerical flexibility.

The values of the different parameters used in this study are as follows:

$$
\begin{aligned}
& \mathrm{C}_{1}=0.49 \mathrm{ca} 1 /{ }^{\circ} \mathrm{C} \mathrm{cm}^{3} \\
& \mathrm{~K}_{1}=9.6 \times 10^{-3} \mathrm{ca} 1 / \mathrm{cm} \mathrm{sec}{ }^{\circ} \mathrm{C} \\
& \mathrm{C}_{2}=0.62 \mathrm{cal} /{ }^{\circ} \mathrm{C} \mathrm{\textrm {cm } ^ { 3 }} \\
& \mathrm{K}_{2}=6.9 \times 10^{-3} \mathrm{cal} / \mathrm{cm} \mathrm{sec}^{\circ} \mathrm{C} \\
& \mathrm{L}=19.2 \mathrm{cla} / \mathrm{cm}^{3} \\
& \mathrm{H}_{\mathrm{S}}=-10^{\circ} \mathrm{C} \\
& \mathrm{H}_{1}=4^{\circ} \mathrm{C}
\end{aligned}
$$

Computed temperature profiles together with the analytic solution as obtained from Ingersoll et al. [7] for zero initial frozen thickness are shown in Figure (2). Twenty Hermitian cubic elements are used in the simulation. The time step in this simulation is continuously adjusted such that the size of the frozen zone increased by a factor of 10 in 15 time step. The temperature in both phases is plotted vs. distance. Steady state solution is obtained after $3 \times 10^{4} \mathrm{sec}$ or 55 time step.
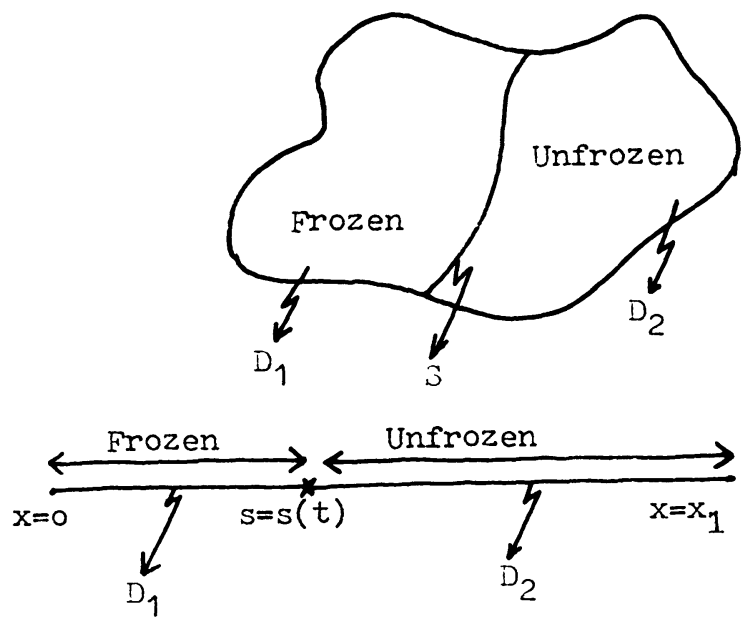

Figure (1). Domain of definition 


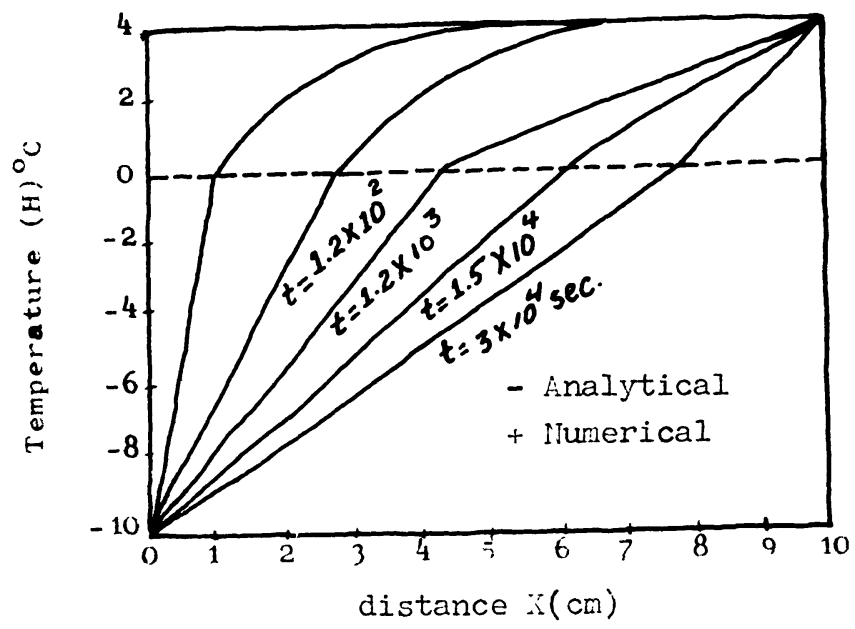

Figure (2). Computed and analytical temperature profile in frozen and unfrozen regions.

5. CONCLUSION.

The present approach for simulation the two-phase Stefan problem is quite general and is applicable to a variety of problems involving moving transition zones and deforming regions. Numerical results obtained by this scheme was accurate and in an excellent agreement with the analytical solutions. This method is valid for one and multi-dimensional situations without any limitation in the formulation, and can be used for simualtion of complicated moving boundary problems.

\section{REFERENCES}

1. HALABI, Y., SHEN, S.H.T., PAPATHEODOROU, T.S., and BRICGS, W.L. "Transpurt and ACrumulation of Frazil Ice Suspensions in Rivers", Mathematical Modelling in sienre and Technology. The Fourth International Conference "urich, (Eds. Xavier drula, J. R., Rundolf E. Kalman, Anthanasios I. Liapis, and Ervin Y. Rodin) Pro\%ram Press, New Y irk, August 1983.

2. Fox, L., "What are the Best Numerical Methods?", in Moving Boundary Problemș iı Heat Flow and Diffusion (Eds. J.R. Ocendon and W.R. Hodgkins), Claredon Press, Oxford, 1975.

3. CRANK, J., "Finite difference methods", in Moving Boundary Problems in Heat Flow and Diffusion 'Eds. J.R. Ocendon and W.R. Hodgkins), Claredon Press, (O)ford, 1975.

4. CRANK, J., and GUPTA, R.S. "Isotherm Migrati n Method in two Dimensions", Int. J. Heat Mass Transf., 18, $1101,1975$.

5. COMINI, G., Del GUIDICE, S., LEWIS, R.W., and ZIENKIEWIC7, D.C. "Finite-Element Solution of Nonlinear Heat Conduction Problens", Int. I. Numerical Methods in Engineerin: $, 8,613(1974)$.

6. MORGAN, K., LEWIS, R.W., and ZIENKIEWLC\%, O.C. "An Improved Algorithm forr Heat conduction Problems with Phase Change", Int. J. Numerical Methods in Engine, 1ing, 12, 1191 (1978).

7. IN':ERSOLL, L.R., 7OBEL, O.J., and INGERSOLL, A.C. "He.lt Conduction", liniv. ut Wisconsin Press, Madisun, 1954. 


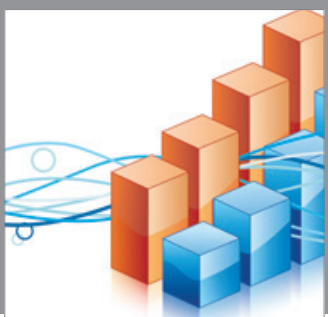

Advances in

Operations Research

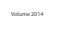

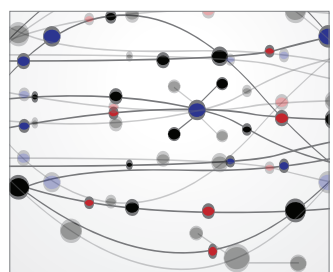

\section{The Scientific} World Journal
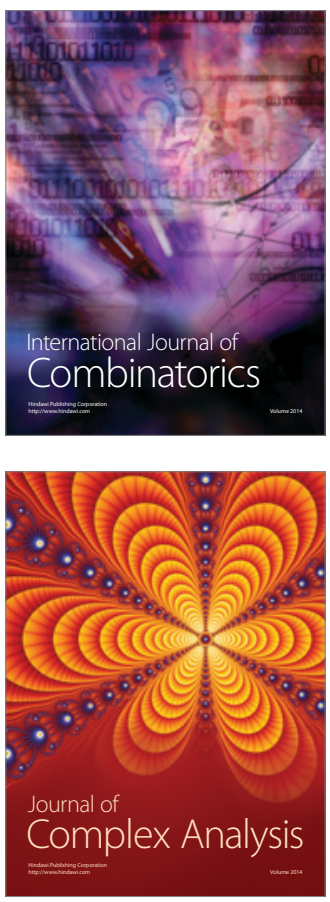

International Journal of

Mathematics and

Mathematical

Sciences
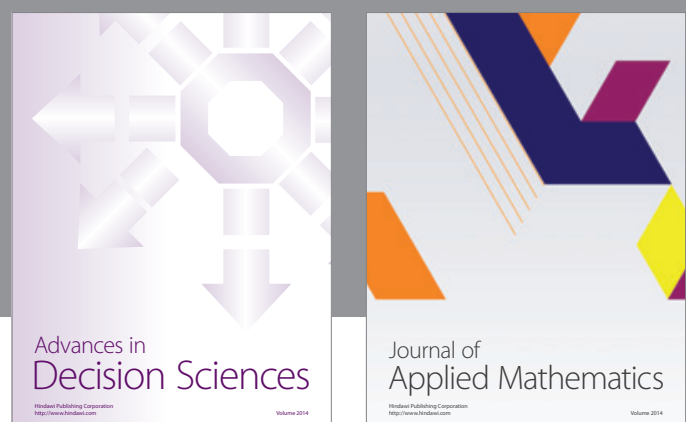

Journal of

Applied Mathematics
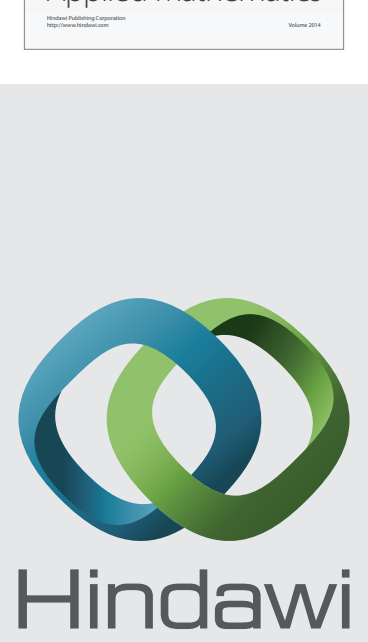

Submit your manuscripts at http://www.hindawi.com
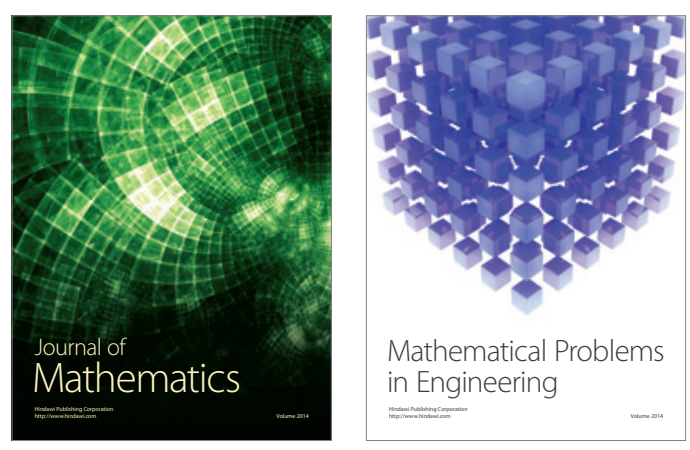

Mathematical Problems in Engineering
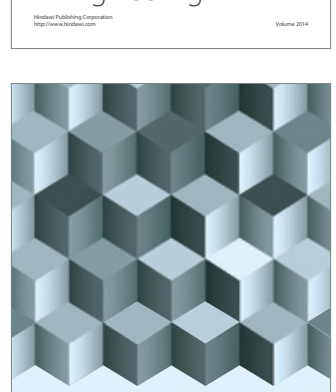

Journal of

Function Spaces
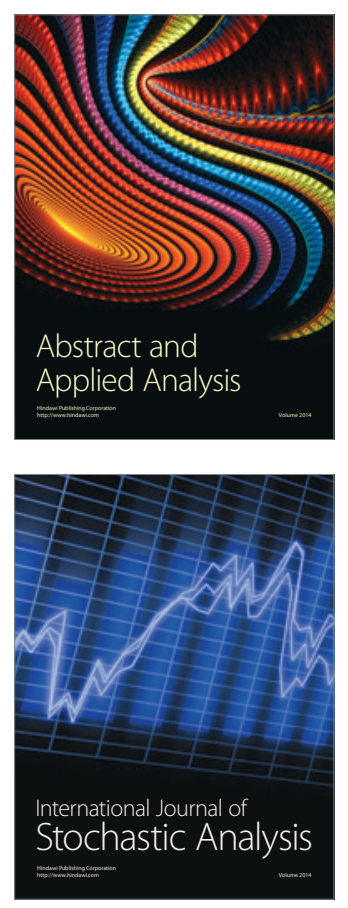

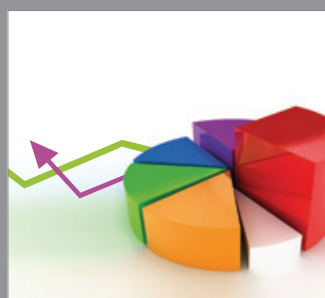

ournal of

Probability and Statistics

Promensencen
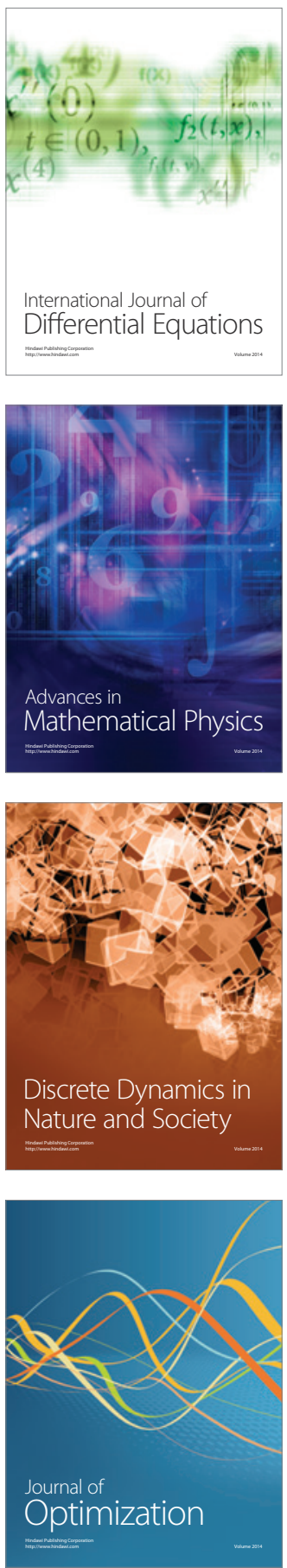